\title{
Classification of integrable discrete Klein-Gordon models
}

\author{
Ismagil T. Habibullin 1 \\ Ufa Institute of Mathematics, Russian Academy of Science, \\ Chernyshevskii Str., 112, Ufa, 450077, Russia \\ Elena V. Gudkova 2 \\ Department of Applied Mathematics and Mechanics, \\ Ufa State Petroleum Technical University, \\ Kosmonavtov str., 1, Ufa, 450062, Russia
}

\begin{abstract}
The Lie algebraic integrability test is applied to the problem of classification of integrable Klein-Gordon type equations on quad-graphs. The list of equations passing the test is presented containing several well-known integrable models. A new integrable example is found, its higher symmetry is presented.
\end{abstract}

Keywords: quad-graph equations, classification, characteristic vector fields, Lie ring, integrability conditions, higher symmetry.

\section{Introduction}

We study the integrability problem for the quad-graph equation of the form

$$
u_{1,1}=f\left(u, u_{1}, \bar{u}_{1}\right)
$$

Such kind equations have a large variety of applications in physics, biology, architecture, etc. Here the unknown $u=u(m, n)$ is a function of two discrete variables $m, n$. For the sake of convenience we use the following notations: $u_{k}=u(m+k, n), \bar{u}_{k}=u(m, n+k), u_{1,1}=$ $u(m+1, n+1)$. Function $f$ is supposed to be locally analytic, it depends essentially on all three arguments. In other words equation (1) can be rewritten in any of the following forms

$$
u_{i, j}=f^{i, j}\left(u, u_{i}, \bar{u}_{j}\right), \quad \text { with } \quad i= \pm 1, j= \pm 1
$$

Nowadays various approaches are known for studying integrable discrete phenomena. The property of consistency around a cube [1], has been proposed as the integrability criterion for quadrilateral difference equations [2], [3], 44. Symmetry approach to the classification of integrable systems is adopted to discrete case [5], [6], [7], [8], [9]. Another characteristic property of an integrable equation is the vanishing of its algebraic entropy [10. Alternative methods are used in [11, [12], 13]. In this article we study discrete phenomena from some different point of view.

Years ago it was observed that characteristic Lie algebras, introduced in [14], in the case of integrable hyperbolic type PDE's like sine-Gordon and Tzitzeica-Zhiber-Shabat equations have a very specific property. The dimensions of the linear spaces spanned by multiple commutators of the generators grow essentially slower than in generic case. In [15] the problem of rigorous

\footnotetext{
${ }^{1} \mathrm{e}$-mail: habibullinismagil@gmail.com

2e-mail: elena.gudkova79@mail.ru
} 
formalization of the notion of "slow growth" has been discussed. A conjecture was suggested and checked by applying to classify integrable equations of the form $u_{x, y}=f\left(u, u_{x}\right)$.

In the recent article [16] we introduced and successfully tested a classification scheme (called algebraic test) based on investigation of multiple commutators of characteristic vector fields defined by equation (11). Now we use the algebraic test (an explanation is given in Section 2 below) to classify discrete Klein-Gordon type equation on quad graph

$$
u_{1,1}+u=g\left(u_{1}+\bar{u}_{1}\right) .
$$

The list of equations which partially passed the algebraic test is studied additionally by applying the symmetry test. It is remarkable that the final list contains in addition to the well-known equations as the discrete potential KdV equation, the discrete Liuoville equation and a discrete analogue of the sine-Gordon equation also a new integrable model (see below the discussion on Corollary of theorem 2 and theorem 3):

$$
u_{1,1}=\frac{\alpha u_{1} \bar{u}_{1}-\beta}{u\left(\alpha+u_{1} \bar{u}_{1}\right)} .
$$

Remind that the problem of complete description of integrable Klein -Gordon equations

$$
u_{x, y}=g(u)
$$

has been solved years ago by A.V.Zhiber and A.B.Shabat [17]. The authors proved that the only integrable equations in (5) are

linear equation;

the Liouville equation $u_{x, y}=e^{u}$;

the sine-Gordon equation $u_{x, y}=\sin u$;

the Tzitzeica-Zhiber-Shabat equation $u_{x, y}=e^{u}+e^{-2 u}$.

The article is organized as follows. In section 2 we define characteristic vector-fields and the test Lie ring, formulate the algebraic test, consider an illustrative example. The classification problem for the model of the form $u_{1,1}+u=g\left(u_{1}+\bar{u}_{1}\right)$ passing the test is investigated in section 3. The result of classification is summarized in Theorems 3 and 5 .

\section{Algebraic test and classification scheme}

Note that for any integers $p$ and $q$ the variable $u_{p, q}$ is expressed in terms of the variables $\left\{u_{i}, \bar{u}_{j}\right\}_{i, j=-\infty}^{\infty}$ in a recurrent way. Hence the variables $u_{i}, \bar{u}_{j}$ are called dynamical variables. They considered as independent ones. For example,

$$
u_{2,1}=f\left(u_{1}, u_{2}, u_{1,1}\right)=f\left(u_{1}, u_{2}, f\left(u, u_{1}, \bar{u}_{1}\right)\right)
$$

and

$$
u_{-1,-2}=f^{-1,-1}\left(\bar{u}_{-1}, u_{-1,-1}, \bar{u}_{-2}\right)=f^{-1,-1}\left(\bar{u}_{-1}, f^{-1,-1}\left(u, u_{-1}, \bar{u}_{-1}\right), \bar{u}_{-2}\right),
$$

Introduce the shift operators $D$ and $\bar{D}$, shifting the first and respectively the second integer arguments: $D h(m, n)=h(m+1, n), \bar{D} h(m, n)=h(m, n+1)$. Explain the action of the operators $D$ and $\bar{D}$ on the functions of dynamical variables. For the function $h=h\left(u_{i}, u_{i-1} \ldots, u_{i^{\prime}}, \bar{u}_{j}, \bar{u}_{j+1}, \ldots, \bar{u}_{j^{\prime}}\right)$ we have

$$
h_{k}=D^{k} h=h\left(u_{i+k}, u_{i+k-1} \ldots, u_{i^{\prime}+k}, \bar{u}_{k, j}, \bar{u}_{k, j+1}, \ldots, \bar{u}_{k, j^{\prime}}\right)
$$


and similarly

$$
\bar{h}_{k}=\bar{D}^{k} h=h\left(u_{i, k}, u_{i, k-1} \ldots, u_{i^{\prime}, k}, \bar{u}_{k+j}, \bar{u}_{k+j+1}, \ldots, \bar{u}_{k+j^{\prime}}\right) .
$$

In these two formulas one has to replace the double shifts $u_{\alpha, \beta}$ through dynamical variables as it is discussed above.

Characteristic vector fields for the hyperbolic type PDE were introduced by E.Goursat [18] in 1899. They provide a very effective tool for classification of Liouville type integrable systems. For instance in [18] a list (almost complete) of hyperbolic type PDE was found admitting integrals in both directions by using a method based on the notion of characteristic vector fields. Interest to the subject renewed after the paper [14], where the exhaustive description of exponential type systems is given admitting complete set of integrals. The concept of characteristic vector fields was adopted to the quad graph equations in [19]. In the recent papers [20], 21], 22] the characteristic vector fields were used for the purpose of classification of semi-discrete Liouville type equations. All of these studies concern with the Liouville type integrability. In [15] and [16] some new applications of these important notions are suggested. Let us remind some necessary definitions. At first suppose that equation (1) admits a non-trivial $n$-integral, i.e. there is a function $I$ depending on a finite number of the dynamical variables $I=I\left(u_{-j}, u_{-j+1}, \ldots u_{k}\right)$ satisfying the equation $\bar{D} I=I$. In an enlarged form the last equation is

$$
I\left(r_{-j+1}, r_{-j+2}, \ldots, r, \bar{u}, f, f_{1}, \ldots f_{k-1}\right)=I\left(u_{-j}, u_{-j+1}, \ldots u_{k}\right),
$$

where the function $r=f^{-1,1}\left(u, u_{-1}, \bar{u}_{1}\right)$ is defined in (2). Since the right hand side of (6) does not depend on the variable $\bar{u}_{1}$ we find $\frac{\partial}{\partial \bar{u}_{1}} \bar{D} I=\frac{\partial}{\partial \bar{u}_{1}} \bar{I}=0$. It implies the equation $Y I=0$ where the operator $Y$ is defined as follows $Y:=\bar{D}^{-1} \frac{\partial}{\partial \bar{u}_{1}} \bar{D}$. Direct computations give (see [16], [19])

$$
Y=\frac{\partial}{\partial u}+x \frac{\partial}{\partial u_{1}}+\frac{1}{x_{-1}} \frac{\partial}{\partial u_{-1}}+x x_{1} \frac{\partial}{\partial u_{2}}+\frac{1}{x_{-1} x_{-2}} \frac{\partial}{\partial u_{-2}}+\ldots
$$

where $x=\bar{D}^{-1}\left(\frac{\partial f\left(u, u_{1}, \bar{u}_{1}\right)}{\partial \bar{u}_{1}}\right)=-\frac{\partial f^{1,-1}\left(u, u_{1}, \bar{u}_{-1}\right) / \partial u}{\partial f^{1,-1}\left(u, u_{1}, \bar{u}_{-1}\right) / \partial u_{1}}$. We call the operators $X:=\frac{\partial}{\partial \bar{u}_{-1}}$ and $Y$ characteristic vector fields. Now it is evident that the map $f\left(u, u_{1}, \bar{u}_{1}\right) \Longrightarrow Y$ is correctly defined for any $f$ due to the formula (7).

Denote through $T$ the set of vector fields obtained by taking all possible multiple commutators of the operators $X$ and $Y$, and taking all linear combinations with coefficients depending on a finite number of the dynamical variables $\bar{u}_{-1}, u, u_{ \pm 1}, u_{ \pm 2}, \ldots$. Evidently the set $T$ has a structure of the Lie ring. We call it test ring of the equation (1) in the direction of $n$. In a similar way one can define the test ring $\bar{T}$ in the direction of $m$.

Notice that for Liouville type integrable equations of the form (1) both $\operatorname{rings} T$ and $\bar{T}$ are of finite dimension. Actually the test ring is a subset of the characteristic Lie ring [19]-[22].

Denote through $V_{j}$ the linear space over the field of locally analytic functions spanned by $X, Y$ and all multiple commutators of $X$ and $Y$ of order less than or equal to $\mathrm{j}$ such that:

$$
V_{0}=\{X, Y\}, \quad V_{1}=\{X, Y,[X, Y]\}, \quad \cdots
$$

Remind that such kind sequences of linear spaces have important applications in geometry 3 (see [23], 24]). Introduce the function $\Delta(k)=\operatorname{dim} V_{k+1}-\operatorname{dim} V_{k}$. The following conjecture is approved by numerous examples.

Conjecture (algebraic test). Any integrable model of the form (1) satisfies the following condition: there is a sequence of natural numbers $\left\{t_{k}\right\}_{k=1}^{\infty}$ such that $\Delta\left(t_{k}\right) \leq 1$.

\footnotetext{
${ }^{3}$ The authors thank E.Ferapontov for drawing their attention to this fact.
} 
Ring $T$ admits an automorphism, generated by the shift operator $D$,

$$
T \ni Z \stackrel{\text { Aut }}{\rightarrow} D Z D^{-1} \in T,
$$

which plays crucial role in our further considerations. It is important that $X$ and $Y$ considered as operators on the set of functions depending on the variables $\bar{u}_{-1}, u, u_{ \pm 1}, u_{ \pm 2}, \ldots$ satisfy the following conjugation relations [16]

$$
D X D^{-1}=p X \quad \text { and } \quad D Y D^{-1}=\frac{1}{x} Y,
$$

where $p=D\left(\frac{\partial f^{-1,-1}\left(u, u_{-1}, \bar{u}_{-1}\right)}{\partial \bar{u}_{-1}}\right)=\frac{1}{\partial f^{1,-1}\left(u, u_{1}, \bar{u}_{-1}\right) / \partial \bar{u}_{-1}}$. Indeed, specify the coefficients of the operator $D X D^{-1}=\sum a_{i} \frac{\partial}{\partial u_{i}}+p \frac{\partial}{\partial \bar{u}_{-1}}$ by applying it to the dynamical variables and find that $a_{i}=D X D^{-1} u_{i}=0$ for any integer $i$. Moreover $p=D X D^{-1} \bar{u}_{-1}=D X f^{-1,-1}\left(u, u_{-1}, \bar{u}_{-1}\right)=$ $D\left(\frac{\partial f^{-1,-1}\left(u, u_{-1}, \bar{u}_{-1}\right)}{\partial \bar{u}_{-1}}\right)$. In a similar way one can prove the second formula. Really apply the operator $D Y D^{-1}=\sum c_{i} \frac{\partial}{\partial u_{i}}+d \frac{\partial}{\partial \bar{u}_{-1}}$ to $u_{i}$ and find $c_{j}=D\left(Y u_{j-1}\right)=Y u_{j}$. Then evaluate $d=$ $D Y D^{-1} \bar{u}_{-1}=f_{u}^{-1,-1}+\frac{1}{x_{-1}} f_{u_{-1}}^{-1,-1}$. Since $u_{-1,-1}=f^{-1,-1}\left(u, u_{-1}, \bar{u}_{-1}\right)$ one gets the equation $u=$ $f^{-1,-1}\left(f\left(u, u_{1}, \bar{u}_{1}\right), u_{1}, \bar{u}_{1}\right)$. Let us differentiate it with respect to $\bar{u}_{1}$ and find $D \bar{D}\left(\frac{\partial f^{-1,-1}}{\partial u}\right) \frac{\partial f}{\partial \bar{u}_{1}}+$ $D \bar{D}\left(\frac{\partial f^{-1,-1}}{\partial u_{-1}}\right)=0$ or the same $\frac{\partial f^{-1,-1}}{\partial u}+\frac{1}{D^{-1} \bar{D}^{-1}\left(\frac{\partial f}{\partial \bar{u}_{1}}\right)} \frac{\partial f^{-1,-1}}{\partial u_{-1}}=0$. Now due to the equation $x=\bar{D}^{-1}\left(\frac{\partial f}{\partial \bar{u}_{1}}\right)$ one concludes that $d=0$.

Lemma 1. Suppose that $Z=\sum_{-\infty}^{\infty} b_{j} \frac{\partial}{\partial u_{j}} \in T$ satisfies the following two conditions:

1) $D Z D^{-1}=c Z$ for some function $c$;

2) $b_{j_{0}} \equiv 0$ for some fixed value of $j=j_{0}$.

Then $Z=0$.

Proof of the Lemma can be found in [16].

Example. As an illustrative example we consider the following discrete Liouville type equation (see [25])

$$
u_{1,1}=\frac{1}{u}\left(u_{1}-1\right)\left(\bar{u}_{1}-1\right) .
$$

Find first an explicit form of the characteristic vector field $Y$. Since $f=\frac{1}{u}\left(u_{1}-1\right)\left(\bar{u}_{1}-1\right)$ and $\frac{\partial f}{\partial \bar{u}_{1}}=\frac{1}{u}\left(u_{1}-1\right)$ then

$$
x=\bar{D}^{-1} \frac{1}{u}\left(u_{1}-1\right)=\frac{1}{\bar{u}_{-1}}\left(u_{1,-1}-1\right) .
$$

Express $u_{1,-1}$ through the dynamical variables due to equation (10). Apply the operator $\bar{D}^{-1}$ to both sides of (10) and find $u_{1}=\frac{1}{\bar{u}_{-1}}\left(u_{1,-1}-1\right)(u-1)$. Comparison of the last equation with (11) yields $x=\frac{u_{1}}{u-1}$, therefore

$$
Y=\frac{\partial}{\partial u}+\frac{u_{1}}{u-1} \frac{\partial}{\partial u_{1}}+\frac{u_{-1}-1}{u} \frac{\partial}{\partial u_{-1}}+\frac{u_{1} u_{2}}{(u-1)\left(u_{1}-1\right.} \frac{\partial}{\partial u_{2}}+\ldots,
$$

For this equation $p=\frac{u-1}{u_{1}}=\frac{1}{x}$, hence $D X D^{-1}=p X, D Y D^{-1}=p Y$. Evaluate $D[X, Y] D^{-1}=$ $p^{2}[X, Y]+p X(p) Y-p Y(p) X$, since $X(p)=Y(p)=0$ we get $D[X, Y] D^{-1}=p^{2}[X, Y]$, moreover $[X, Y]=\sum_{j=1}^{\infty} a_{j} \frac{\partial}{\partial u_{j}}+a_{-j} \frac{\partial}{\partial u_{-j}}$. Now due to the Lemma 1 one obtains $[X, Y]=0$. So dimension of the ring $T$ for equation (10) equals two and $\Delta(k)=0$ for all $k \geq 0$ therefore equation (10) passes the test. 


\section{Equations of the form $u_{1,1}+u=g\left(u_{1}+\bar{u}_{1}\right)$}

In this section we apply the Conjecture to the following Klein-Gordon type particular class of discrete model (1)

$$
u_{1,1}+u=g\left(u_{1}+\bar{u}_{1}\right) .
$$

where the function $g$ is to be determined.

Classification scheme. Obviously equation (11) passing the algebraic test above should satisfy one of the conditions:

i) $\Delta(0)<\Delta_{\max }(0)=1$

ii) $\Delta(0)=\Delta_{\max }(0), \Delta(1)<\Delta_{\max }(1)=2$;

iii) $\Delta(0)=\Delta_{\max }(0), \Delta(1)=\Delta_{\max }(1), \Delta(2)<\Delta_{\max }(2)=3$;

iv) $\Delta(0)=\Delta_{\max }(0), \Delta(1)=\Delta_{\max }(1), \Delta(2)=\Delta_{\max }(2)$ and $\Delta(k) \leq 1$ for some $k>2$.

Where $\Delta_{\max }(k)$ stands for the greatest value of $\Delta(k)$ for equation (11) when $f\left(u, u_{1}, \bar{u}_{1}\right)$ ranges the class of arbitrary functions.

Remark that in the case of equation (13) investigation of the first three particular cases i)-iii) allows one to extract a very short list of equations expected to be integrable. The list is exhaustive because the case $i v$ ) is never realized (see below Corollaries of Theorems 2 and 4 ).

Introduce vector fields $R_{1}=[X, Y], P_{1}=\left[X, R_{1}\right], Q_{1}=\left[Y, R_{1}\right], R_{2}=\left[X, Q_{1}\right], W=\left[Y, Q_{1}\right]$, $Z=\left[X, P_{1}\right]$. Using these vector fields we can span in addition to $V_{0}$ and $V_{1}$ two more linear spaces:

$$
V_{2}=V_{1}+\left\{P_{1}, Q_{1}\right\}, \quad V_{3}=V_{2}+\left\{W, Z, R_{2}\right\} .
$$

In order to evaluate $\Delta(k)$ we will use the automorphism (8) . At first evaluate the factors $x$ and $p$ in formula (9) for the case (13). We have $x=p=g^{\prime}\left(g^{-1}\left(u_{1}+\bar{u}_{-1}\right)\right)$, where the function $\beta=g^{-1}(\alpha)$ is the inverse to the function $\alpha=g(\beta)$. Inversely, knowing $x=x\left(u_{1}+\bar{u}_{-1}\right)$ one can recover $g(\beta)$ by using the equation

$$
\beta=g^{-1}(\alpha)=\int\left(g^{-1}(\alpha)\right)^{\prime} d \alpha=\int \frac{d \alpha}{g^{\prime}\left(g^{-1}(\alpha)\right.}=\int \frac{d \alpha}{x(\alpha)} .
$$

Specify the action of the characteristic operators $X$ and $Y$ on the variable $x$. Evidently, $X x=x^{\prime}, Y x=x x^{\prime}$. It is found by direct calculation that

$$
\begin{aligned}
D R_{1} D^{-1} & =R_{1}-\frac{x^{\prime}}{x} Y-x^{\prime} X, \\
D P_{1} D^{-1} & =x P_{1}-x^{\prime} R_{1}-r Y-x r X, \quad r=x^{\prime \prime}-\frac{x^{\prime 2}}{x}, \\
D Q_{1} D^{-1} & =\frac{1}{x} Q_{1}+\frac{x^{\prime}}{x} R_{1}-\frac{x^{\prime \prime}}{x} Y-x^{\prime \prime} X \\
D W D^{-1} & =\frac{1}{x^{2}} W+\left(\frac{2 x^{\prime \prime}}{x}-\frac{x^{\prime 2}}{x^{2}}\right) R_{1}-\frac{x^{\prime \prime \prime}}{x} Y-x^{\prime \prime \prime} X, \\
D Z D^{-1} & =x^{2} Z+\left(x^{\prime 2}-2 x x^{\prime \prime}\right) R_{1}-q Y-x q X, \quad q=x x^{\prime \prime \prime}-2 x^{\prime} x^{\prime \prime}+\frac{x^{\prime 3}}{x}, \\
D R_{2} D^{-1} & =R_{2}-\frac{x^{\prime}}{x} Q_{1}+x^{\prime} P_{1}-\frac{x^{\prime 2}}{x} R_{1}-s Y-x s X, \quad s=x^{\prime \prime \prime}-\frac{x^{\prime} x^{\prime \prime}}{x},
\end{aligned}
$$


Let us study the set $G$ of all multiple commutators of $X$ and $Y$.

Lemma 2. The coefficients of any operator in $G$ are functions of a finite number of the dynamical variables $x, x_{ \pm 1}, x_{ \pm 2}, \ldots$.

Proof. Here $x(\alpha)$ is a fixed function of the argument $\alpha=u_{1}+\bar{u}_{-1}$. Therefore one can write $x^{\prime}=\phi(x)$ for some function $\phi$. Then $X(x)=-\phi(x)$ and $Y(x)=x \phi(x)=: \psi(x)$. By using the conjugation relations $D X D^{-1}=x X$ and $D Y D^{-1}=\frac{1}{x} Y$ one derives that $X\left(x_{j}\right)=$ $\phi^{j}\left(x, x_{1}, \ldots x_{j}\right)$ and $Y\left(x_{j}\right)=\psi^{j}\left(x, x_{1}, \ldots x_{j}\right)$. Similarly $X\left(x_{-j}\right)=\phi^{-j}\left(x, x_{-1}, \ldots x_{-j}\right)$ and $Y\left(x_{-j}\right)=$ $\psi^{-j}\left(x, x_{-1}, \ldots x_{-j}\right)$. Now evidently $R_{1}=X(x) \frac{\partial}{\partial u_{1}}+X\left(\frac{1}{x_{-1}}\right) \frac{\partial}{\partial u_{-1}}+X\left(x x_{1}\right) \frac{\partial}{\partial u_{2}}+\cdots$ satisfies the statement of the lemma. Due to the formulas $R_{1}(x)=X(x) x^{\prime}$ and $D R_{1} D^{-1}=R_{1}+\frac{x^{\prime}}{x} Y-x^{\prime} X$ one gets $R_{1}\left(x_{j}\right)=\phi^{j}\left(x, x_{1}, \ldots x_{j}\right)$. Obviously the proof can be completed by using induction.

Theorem 2. Suppose that an equation of the form (13) satisfies one of the conditions $i)$ - iii) of the Classification scheme then function $x=x(\alpha)$ solves the following ordinary differential equation

$$
x^{\prime 2}=\left(x^{2}+1\right) \gamma+x \nu
$$

with the constant coefficients $\gamma, \nu$.

Proof of the theorem 2. Begin with the case $i$ ), suppose that $\Delta(0)=0$, then we have $R_{1}=\lambda X+\mu Y$. It is evident that $R_{1}=X(x) \frac{\partial}{\partial u_{1}}+\ldots, X=\frac{\partial}{\partial \bar{u}_{-1}}$ and $Y=\frac{\partial}{\partial u}+x \frac{\partial}{\partial u_{1}}+\ldots$ hence $\lambda=\mu=0$ and therefore $R_{1}=0$. By applying the automorphism above to both sides of the last equation one finds

$$
-\frac{x^{\prime}}{x} Y-x^{\prime} X=0
$$

Since $X$ and $Y$ are linearly independent we get equation $x^{\prime}=0$ which is a particular case of (16). Evidently its solution is $x=c$ and due to (14) it can be found that $\beta=g^{-1}(\alpha)=\frac{1}{c} \alpha+c_{1}$. Thus our equation $\alpha=g(\beta)$ (see (13)) is linear $u_{1,1}+u=c\left(u_{1}+\bar{u}_{1}+c_{1}\right)$. For this case $\operatorname{dim} T=2$ so that $\Delta(k)=0$ for $k \geq 0$. In a similar way one checks that condition $i i$ ) leads to (16). Indeed suppose that $\Delta(0)=1$ and $\Delta(1)<2$ then we have

$$
P_{1}=\nu Q_{1}+\epsilon R_{1} .
$$

Here due to Lemma 2 functions $\nu$ and $\epsilon$ might depend only on $x, x_{ \pm 1}, x_{ \pm 2} \ldots$ Apply the automorphism (8) to both sides of (17) then simplify due to equations (15):

$x\left(\nu Q_{1}+\epsilon R_{1}\right)-x^{\prime} R_{1}-r Y-x r X=D(\nu)\left(\frac{1}{x} Q_{1}+\frac{x^{\prime}}{x} R_{1}-\frac{x^{\prime \prime}}{x} Y-x^{\prime \prime} X\right)+D(\epsilon)\left(R_{1}-\frac{x^{\prime}}{x} Y-x^{\prime} X\right)$.

Comparison of the coefficients before linearly independent operators gives rise to the conditions

$$
\begin{array}{ll}
Q_{1}: & x \nu=\frac{1}{x} D(\nu) \\
R_{1}: & -x^{\prime}+x \epsilon=\frac{x^{\prime}}{x} D(\nu)+D(\epsilon) \\
Y: & -r=-\frac{x^{\prime}}{x} D(\epsilon)+\frac{x^{\prime \prime}}{x} D(\nu) ; \\
X: & -x r=-x^{\prime} D(\epsilon)-x^{\prime \prime} D(\nu) .
\end{array}
$$

Simple analysis of these equations implies that equation (17) holds if and only if the following three conditions valid: $\nu=0, \epsilon=$ const, $x^{\prime}=\epsilon(x-1)$. Actually, under these conditions the last two equations above are satisfied automatically. In a similar way one can check that $Q_{1}=\nu P_{1}-\epsilon R_{1}$ is equivalent to the same three conditions. Hence if $\Delta(1)<2$ then $\Delta(1)=0$ and consequently $\Delta(k)=0$ for any natural $k \geq 1$. Thus in this case $\operatorname{dim} T=3$. 
Let us suppose now that $\Delta(0)=1, \Delta(1)=2$ and $\Delta(2) \leq 2$ which corresponds to the case iii). At first consider the case when $Z$ is linearly expressed through the other vector fields in subspace $V_{3}$ :

$$
Z=\gamma R_{1}+\delta P_{1}+\epsilon Q_{1}+\phi R_{2}+\psi W .
$$

Apply the automorphism (8) to both sides of equation (18) and compare coefficients before linearly independent operators

$$
\begin{aligned}
W: & x^{2} \psi=D(\psi) \frac{1}{x^{2}} \\
R_{2}: & x^{2} \phi=D(\phi) \\
Q_{1}: & x^{2} \epsilon=D(\epsilon) \frac{1}{x}+\frac{x^{\prime}}{x} \phi \\
P_{1}: & x^{2} \delta=D(\delta) x+x^{\prime} \phi \\
R_{1}: & x^{2} \gamma=x^{\prime 2}-2 x x^{\prime \prime}=D(\delta) x^{\prime}+D(\gamma) .
\end{aligned}
$$

Since $x=x\left(u_{1}+\bar{u}_{-1}\right)$ we have $\psi=0, \phi=0, \epsilon=0, \delta=0, \gamma=$ const. Comparison of the coefficients of $X$ and $Y$ gives one more equation $x q=\gamma x^{\prime}$. Finally we get two ordinary differential equations for $x$ which are absolutely the same as in the case of the equation $u_{1,1}-u=$ $g\left(u_{1}-\bar{u}_{1}\right)$ (see [16]):

$$
x^{2} x^{\prime \prime \prime}-2 x x^{\prime} x^{\prime \prime}+x^{\prime 3}=\gamma x^{\prime}, \quad\left(x^{2}-1\right) \gamma+x^{\prime 2}-2 x x^{\prime \prime}=0 .
$$

The compatibility condition of these equations is equivalent to equation (16). In this case we have $Z=\gamma R_{1}$. It is remarkable that $x$ solves equation (16) if and only if $W$ is linearly expressed through the other elements of $V_{3}$ and then $W=\gamma R_{1}$. And the last possibility is when $R_{2}$ is linearly expressed through $X, Y, R_{1}, P_{1}, Q_{1}, W, Z$. In this case $x$ solves the equation $x^{\prime}=0$. The proof of the theorem is completed.

In order to find $x=x(\alpha)$ evaluate the integral

$$
H(x):=\int \frac{d x}{\sqrt{\left(x^{2}+1\right) \gamma+x \nu}}=\alpha-\alpha_{0} .
$$

For the case $\gamma \neq 0$ the answer is given by the formula

$$
H(x)=\frac{1}{\sqrt{\gamma}} \ln \left(2 \sqrt{x^{2}+1+x b}+2 x+b\right), \quad b=\frac{\nu}{\gamma} .
$$

Now find $x$ by solving the equation $H(x)=\alpha-\alpha_{0}$.

$$
x(\alpha)=\frac{1}{4} e^{\sqrt{\gamma}\left(\alpha-\alpha_{0}\right)}-\frac{\nu}{2 \gamma}-\left(1-\frac{\nu^{2}}{4 \gamma^{2}}\right) e^{-\sqrt{\gamma}\left(\alpha-\alpha_{0}\right)} .
$$

In order to get the corresponding quad-graph equation (13) integrate again:

$$
\beta=g^{-1}(\alpha)=\int \frac{d \alpha}{x(\alpha)} .
$$

Integration gives

$$
\beta=\frac{1}{\sqrt{\gamma}} \ln \left|\frac{e^{\sqrt{\gamma}\left(\alpha-\alpha_{0}\right)}-b-2}{e^{\sqrt{\gamma}\left(\alpha-\alpha_{0}\right)}-b+2}\right|+\beta_{0} .
$$


Then the equation searched is given by the formula (take $\sqrt{\gamma}=1$ )

$$
a_{1} e^{u_{1,1}+u_{1}+\bar{u}_{1}+u}+a_{2} e^{u_{1,1}+u}+a_{3} e^{u_{1}+\bar{u}_{1}}+a_{4}=0,
$$

where $a_{1}=e^{-\alpha_{0}-\beta_{0}}, a_{2}=-e^{-\alpha_{0}}, a_{3}=(2-b) e^{-\beta_{0}}, a_{4}=2+b$. By setting $u=\ln v$ one reduces it to a quadrilinear form

$$
a_{1} v_{1,1} v_{1} \bar{v}_{1} v+a_{2} v_{1,1} v+a_{3} v_{1} \bar{v}_{1}+a_{4}=0
$$

with arbitrary constants $a_{j}$.

If $\gamma=0$, then $x=x(\alpha)$ solves the equation $x^{\prime}=\sqrt{\nu x}$. Thus obviously $x=c\left(\alpha-\alpha_{0}\right)^{2}$ and therefore

$$
\beta=g^{-1}(\alpha)=\int \frac{d \alpha}{c\left(\alpha-\alpha_{0}\right)^{2}}=\frac{d}{\alpha-\alpha_{0}}-\beta_{0}, d=-1 / c .
$$

The corresponding equation (13) follows from $\left(\alpha-\alpha_{0}\right)\left(\beta-\beta_{0}\right)=d$. It is of the form

$$
\left(u_{1,1}+u-\alpha_{0}\right)\left(u_{1}+\bar{u}_{1}-\beta_{0}\right)=d .
$$

Corollary of Theorem 2. If for a nonlinear chain (13) one of the conditions $i$ ) - iii) is satisfied then the chain is of one of the form:

1) $u_{1,1}+u=c\left(u_{1}+\bar{u}_{1}+c_{1}\right)$;

2) $a_{1} u_{1,1} u_{1} \bar{u}_{1} u+a_{2} u_{1,1} u+a_{3} u_{1} \bar{u}_{1}+a_{4}=0$;

3) $\left(u_{1,1}+u-\alpha_{0}\right)\left(u_{1}+\bar{u}_{1}-\beta_{0}\right)=d$.

Let us study in details the list obtained. It is remarkable that it contains a new integrable example of equation (11).

Theorem 3. The chain 2) in the Corollary of Theorem 2 admits a symmetry of the form

$$
u_{t}=g\left(u_{1}, u_{-1}, u, \bar{u}_{1}, \bar{u}_{-1}\right)
$$

if and only if the condition holds $k:=\frac{a_{3}}{a_{2}}= \pm 1$. Under this condition the symmetry is of the form

$$
u_{t}=\lambda u \frac{k u_{1}+u_{-1}}{k u_{1}-u_{-1}}+\mu \bar{u} \frac{k \bar{u}_{1}+\bar{u}_{-1}}{k \bar{u}_{1}-\bar{u}_{-1}} .
$$

Chain 3) admits a symmetry of the form (25) if and only if $\alpha_{0}=\beta_{0}$ and then it is reduced to the discrete potential KdV equation.

Theorem can be proved by using technique developed in [5]. Actually it claims that the chain 2) with $a_{3}= \pm a_{2}$ passes the symmetry test. For the case $k=1$ (or $a_{3}=a_{2}$ ) the chain is a particular case of the Viallet equation (see [10]), this means that by a point transformation it can be reduced to one of the equations of ABS list [4. Higher symmetries for this case are found in [6]. For some special choice of the parameters chain 2) with $k=-1$ is reduced to the discrete sin-Gordon equation

$$
3\left(u_{1,1} u_{1} \bar{u}_{1} u-1\right)+u_{1,1} u-u_{1} \bar{u}_{1}=0
$$

found by R. Hernandez Heredero and C. Scimiterna. Recently in [5] it was proved that equation (26) admits higher symmetries. To the best of our knowledge general case of the equation 2) with $k=-1$ has never been identified before as integrable one. 
When $a_{1}=0$ and $a_{3}=-a_{2}$ it is reduced to the well-known discrete Liouville equation

$$
e^{u_{1,1}+u}=e^{u_{1}+\bar{u}_{1}}+1
$$

having nontrivial integrals, i.e. functions solving the equations $\bar{D} F=F$ and $D \bar{F}=\bar{F}$, with $F=e^{u-u_{1}}+e^{u_{2}-u_{1}}$ and $\bar{F}=e^{u-\bar{u}_{1}}+e^{\bar{u}_{2}-\bar{u}_{1}}$.

The second part of the theorem claims that case 3) admits a symmetry of small order only if it coincides up to a point transformation with the discrete pkdv equation. The question is still open whether it admits any symmetry of a more complicated form, say $u_{t}=$ $g\left(u_{2}, u_{1}, u_{-1}, u, \bar{u}_{1}, \bar{u}_{-1}, \bar{u}_{-2}\right)$. There are some technical difficulties with applications of the symmetry approach to discrete models (see discussion in [5]).

Turn back to the set $G$ consisting of $X, Y$ and their all multiple commutators. Assign two integers: order and degree to each element in $G$. Define the order of an element $Z \in G$ as a number of its factors $X$ and $Y$ minus one. For instance $\operatorname{ord}[X, Y]=1$, ord $[X,[X, Y]]=2$ and so on. Define the degree $\operatorname{deg}(Z)$ of $Z$ as the exponent $k$ in the expansion of the operator obtained by applying the automorphism (8) : $D Z D^{-1}=x^{k} Z+\ldots$, where the tail is a linear combination of the elements with order less than $\operatorname{ord}(Z)$. Denote through $G_{i, j}$ a subset of $G$ containing elements with the order $i$ and the degree $j$. Let $G_{i}=\cup_{j} G_{i, j}$ be the union of all $G_{i, j}$ with one and the same $i$. Evidently the set $G_{i, i-1}$ (as well as $G_{i,-i+1}$ ) contains the only element $Z_{i, i-1}=a d_{X}^{i}(Y)$ up to the factor -1 (correspondingly, the only element $Z_{i,-i+1}=a d_{Y}^{i}(X)$ up to the factor -1$)$. Here the operator $a d$ is defined as follows $a d_{X}(Y)=[X, Y]$.

Theorem 4. Suppose that $Z_{k, k-1}$ (or $\left.Z_{k,-k+1}\right)$ is in the basis of the linear space $V_{k} \supset G_{k}$ for any integer $k$ : $3 \leq k<N$, but $Z_{N, N-1}$ (respectively $Z_{N,-N+1}$ ) is linearly expressed through the other operators in $V_{N}$ then function $x=x\left(u_{1}+\bar{u}_{-1}\right)$ solves an equation of the form $x^{\prime}=\epsilon(x-1)$ with the constant coefficient $\epsilon$.

The proof of the theorem is done exactly in the same way as the proof of Theorem 3 from [16], but presented in the article for the readers' convenience.

Lemma 3. For any integer $k \geq 3$ we have

$$
D Z_{k+1, k} D^{-1}=x^{k} Z_{k+1, k}+c_{k} x^{\prime} x^{k-1} Z_{k, k-1}+\cdots,
$$

where $c_{k} \geq 0$ (but $c_{k}>0$ for $k>3$ ) and the tail contains a linear combination of the operators with the order less than $k$.

Prove the Lemma 3 by induction. From the list of equations (15) one gets for $Z=Z_{3,2}$ and $R_{1}=Z_{1,0}$ the following representation

$$
D Z_{3,2} D^{-1}=x^{2} Z_{3,2}+\left(x^{\prime 2}-2 x x^{\prime \prime}\right) Z_{1,0}-q Y-x q X, \quad q=x x^{\prime \prime \prime}-2 x^{\prime} x^{\prime \prime}+\frac{x^{\prime 3}}{x}
$$

showing that the statement is true for the case $k=3$. Suppose now that $D Z_{k, k-1} D^{-1}=$ $x^{k-1} Z_{k, k-1}+c_{k-1} x^{\prime} x^{k-2} Z_{k-1, k-2}+\cdots$ and evaluate $D Z_{k+1, k} D^{-1}$ :

$$
D Z_{k+1, k} D^{-1}=\left[x X, x^{k-1} Z_{k, k-1}+c_{k-1} x^{\prime} x^{k-2} Z_{k-1, k-2}+\cdots\right]=x^{k} Z_{k+1, k}+c_{k} x^{\prime} x^{k-1} Z_{k, k-1}+\cdots
$$

where $c_{k}=c_{k-1}+(k-1)>c_{k-1} \geq 0$. The proof is completed.

Proof of the Theorem 4. Suppose that

$$
Z_{N, N-1}=\sum_{\operatorname{ord}\left(Z_{\nu}\right)=N} a_{\nu} Z_{\nu}+\sum_{\operatorname{ord}\left(Z_{\mu}\right)=N-1} b_{\mu} Z_{\mu}+\cdots
$$


where $Z_{\nu}$ and $Z_{\mu}$ range the basis of $V_{N}$ and the tail contains a linear combination of the operators of less order. Apply the automorphism (8) to both sides of (29):

$$
\begin{aligned}
& x^{N-1}\left(\sum_{\operatorname{ord}\left(Z_{\nu}\right)=N} a_{\nu} Z_{\nu}+\sum_{\operatorname{ord}\left(Z_{\mu}\right)=N-1} b_{\mu} Z_{\mu}+\cdots\right)-x^{N-2} x^{\prime} c_{N-1} Z_{N-1, N-2}+\cdots= \\
& =\sum_{\operatorname{ord}\left(Z_{\nu}\right)=N} D\left(a_{\nu}\right)\left(x^{k_{\nu}} Z_{\nu}+\cdots\right)+\sum_{\operatorname{ord}\left(Z_{\mu}\right)=N-1} D\left(b_{\mu}\right)\left(x^{k_{\mu}} Z_{\mu}+\cdots\right) .
\end{aligned}
$$

Collect the coefficients before $Z_{\nu}$ and get

$$
x^{N-1} a_{\nu}=x^{k_{\nu}} D\left(a_{\nu}\right), \quad k_{\nu} \neq N-1 .
$$

Due to Lemma 2 functions $a_{\nu}$ and $b_{\mu}$ depend on $x$ and its shifts. Moreover it follows from (30) that $a_{\nu}$ cannot depend on $x, x_{ \pm 1}, x_{ \pm 2}, \ldots$ at all. Therefore the only possibility is $a_{\nu}=0$. Compare now the coefficients before $Z_{N-1, N-2}$ and find:

$$
x^{N-1} b-c_{N-1} x^{N-2} x^{\prime}=x^{N-2} D(b),
$$

where $b$ is the coefficient of $Z_{N-1, N-2}$ in the expansion (29). A simple analysis of equation (31) shows that $b$ is constant. Thus (31) is equivalent to the equation $x^{\prime}=\epsilon(x-1)$ with $\epsilon=b / c_{N-1}$.

Corollary of Theorem 4 . The case iv) of the Classification scheme is never realized.

Proof. Suppose on contrary that such a case is realized. Then at least one of the vector fields $Z_{k, k-1}$ or $Z_{k,-k+1}$ should be linearly expressed through other elements of $V_{k}$, otherwise $\Delta(k) \geq 2$. Therefore due to Theorem 4 we have $x^{\prime}=\epsilon(x-1)$ which corresponds to the case $\left.i i\right)$ $\Delta(0)=1, \Delta(1)<2$ and $\operatorname{dim} T=3$. The contradiction shows that our assumption is not true. The proof is completed.

Let us summarize the results of reasonings above in the following theorem.

Theorem 5. Suppose that an equation of the form (13) passes the algebraic test then it is of one of the form given in Corollary of Theorem 2.

\section{Conclusions.}

A classification scheme introduced recently in [16] is applied to quad-graph Klein-Gordon type equation. The list of equations passed the test contains along with the well-known integrable models some new example which passes also the symmetry test.

\section{Acknowledgments}

The authors thank Prof. A.V.Zhiber and Dr. R.N.Garifullin for valuable advises. This work is partially supported by Russian Foundation for Basic Research (RFBR) grants \# 10-01-91222CT-a, \# 11-01-00732-a, \# 11-01-97005-r-povoljie-a, and \# 10-01-00088-a.

\section{References}

[1] Nijhoff, F. W., Walker, A. J.: The discrete and continuous Painleve hierarchy and the Garnier system. Glasgow Math. J. 43A, 109123 (2001)

[2] Bobenko, A. I., Suris, Yu. B.: Integrable systems on quad-graphs. Int. Math. Res. Notices 11, $573611(2002)$ 
[3] Nijhoff, F. W.: Lax pair for the Adler (lattice Krichever-Novikov) system. Phys. Lett. A 297, $4958(2002)$

[4] V.E. Adler, A.I. Bobenko, Yu.B. Suris. Classification of integrable equations on quadgraphs. The consistency approach. Comm. Math. Phys. 233 (2003) 513543.

[5] Levi D. and Yamilov R.I., Generalized symmetry integrability test for discrete equations on the square lattice. Submitted to JPA. arXiv:1011.0070v1[nlin.SI].

[6] Xenitidis, P.: Integrability and symmetries of difference equations: the AdlerBobenkoSuris case. In Proceedings of the 4th Workshop Group Analysis of Differential Equations and Integrable Systems (2009) arXiv: 0902.3954.

[7] Rasin, O. G., Hydon, P. E.: Conservation laws for integrable difference equations. J. Phys. A: Math. Theor. 40, 1276312773 (2007)

[8] Alexander V. Mikhailov, Jing Ping Wang and Pavlos Xenitidis, Recursion operators, conservation laws and integrability conditions for difference equations, arXiv : 1004.5346v1[nlin.SI]

[9] A. Tongas, D. Tsoubelis, and P. Xenitidis, A family of integrable nonlinear equations of hyperbolic type J. Math. Phys. 42, 5762 (2001).

[10] Bellon, M. P., Viallet, C. M.: Algebraic entropy. Comm. Math. Phys. 204, 425437 (1999)

[11] Nijhoff, F. W., Ramani, A., Grammaticos, B., and Ohta, Y. On discrete Painleve equations associated with the lattice KdV systems and the Painleve VI equation. Stud. Appl. Math. 106, 3 (2001), 261314.

[12] B. Grammaticos, G. Karra, V. Papageorgiou, A. Ramani, Integrability of discrete-time systems, Chaotic dynamics, (Patras,1991), NATO Adv. Sci. Inst. Ser. B Phys. , 298, 75-90, Plenum, New York, (1992).

[13] J. Hietarinta. Searching for CAC-maps. J. Nonl. Math. Phys. 12:2 suppl. (2005) 223230.

[14] A. N. Leznov, V. G. Smirnov, A. B. Shabat, Group of inner symmetries and integrability conditions for two-dimensional dynamical systems, Teoret. Mat. Fizika, 51, no:1, 10-21 (1982).

[15] A. V. Zhiber, R. D. Murtazina, On the characteristic Lie algebras for the equations $u_{x y}=f\left(u, u_{x}\right)$, (In Russian), Fundam. Prikl. Mat.,12, no. 7,65-78 (2006).

[16] Ismagil T. Habibullin, Elena V. Gudkova, An algebraic method of classification of $S$ integrable discrete models arXiv:1006.3423v2[nlin.SI], 27 Oct 2010

[17] A.V.Zhiber, A.B.Shabat, Klein-Gordon equations with a nontrivial group, Soviet Physics Doklady, Vol. 24, p.607

[18] E. Goursat, Recherches sur quelques équations aux dérivées partielles du second ordre, Annales de la faculté des Sciences de l'Université de Toulouse $2^{e}$ série, tome $1, \mathrm{n}^{0} 1$ (1899) p.31-78. 
[19] I. T. Habibullin, Characteristic algebras of fully discrete hyperbolic type equations, Symmetry, Integrability and Geometry: Methods and Applications, no. 1, paper 023, 9 pages, (2005) // arxiv : nlin.SI/0506027.

[20] I. Habibullin, A. Pekcan, Characteristic Lie Algebra and Classification of Semi-Discrete Models, Theoret. and Math. Phys., 151, no. 3, 781-790 (2007) //arXiv:nlin/0610074.

[21] I. Habibullin, N. Zheltukhina, A. Pekcan, On the classification of Darboux integrable chains, Journal of Math. Phys., 49, Issue: 10, 102702 (2008) //arXiv : nlin/0806.3144.

[22] I. Habibullin, N. Zheltukhina, A. Pekcan, Complete list of Darboux integrable chains of the form $t_{1 x}=t_{x}+d\left(t, t_{1}\right)$. Journal of Math. Phys., 50, 102710 (2009). //arXiv : 0907.3785.

[23] E. Cartan, Les systemes de Pfaff a cinque variables et les equations aux derivees partielles du second ordre, Oeuvres completes, Partie II, vol.2, Paris, Gautier-Villars, 1953, 927-1010.

[24] Boris Doubrov, Igor Zelenko, On local geometry of nonholonomic rank 2 distributions arXiv:math/0703662v1[math.DG]

[25] V. E. Adler, S. Ya. Startsev, On discrete analogues of the Liouville equation, Teoret. Mat. Fizika, 121, no. 2, 271-284 (1999), (English translation: Theoret. and Math. Phys., 121, no. 2, 1484-1495, (1999)). 